\title{
A novel mathematical tool for generating highly conserved protein domain viadifferent organismal genomic landscapes
}

\author{
Arunava Goswami ${ }^{\text {, }}$ PabitraPal Choudhury $\#$, Rajneesh Singh ${ }^{\#}$, Sk. SarifHassan ${ }^{\text {, \# }}$ \\ \$BiologicalSciences Division and "Applied StatisticsUnit, Indian StatisticalInstitute,203, B. T. \\ Road, Kolkata 700108 , India
}

Darwinian evolution hypothesizes that a short stretch of DNA was first constructed and then itexpanded to give rise to a long strand. This long strand then produced a mix of exons, introns and repetitiveDNA sequence. The order of production of above three kinds of DNA sequence isunknown. Reshuffling of stretches of DNA like above within organisms has given rise to different chromosomes. Tilldate it is not known how this process is governed. In this paper we show that starting with a sixteen base-pair human olfactory DNA sequence one can form a highly conserved protein domain. Once thisdomain is formed repetitive DNA sequences of a particularkind starts generating which signifies that this particularconserved protein domain willbe unique in nature. The entire mathematical exercise presented in this paper is based on simplest possible context free L-System which we think has been adopted by biological system ingeneral.

Context free L-System [1, 2, 3, 4], considered to be one of simplest, originallyproposed by Hungarian BiologistA. Lindenmayer [5] to study symmetry of plants can be used to generate a large length of DNA sequence with a definite size limit.We took a human olfactory receptor DNA sequence (OR1F1) and derived an L-System with following production rule [ (Axiom: A) A ATGA, C GCGG, T GACA, G AACC] which basicallycovers extreme 5'end of OR 1 F 1.1024 bp length DNA sequence [Fig. 1] generated from this production rule showed 3 Open Reading Frames (ORF) in 3' 5' direction [Fig.2].

Fig. 1

ATGAGACAAACCATGAAACCATGAGCGGATGAATGAATGAGCGGGCGGATGAGACAAACCATGAATGAATGAGCGGGCGGATGAGACAAACCATGAAACCGCGGAACCAAC CATGAGACAAACCATGAATGAGACAAACCATGAATGAGACAAACCATGAAACCGCGGAACCAACCAACCGCGGAACCAACCATGAGACAAACCATGAAACCATGAGCGGAT GAATGAATGAGCGGGCGGATGAGACAAACCATGAATGAGACAAACCATGAATGAGACAAACCATGAAACCGCGGAACCAACCAACCGCGGAACCAACCATGAGACAAACCA TGAAACCATGAGCGGATGAATGAATGAGCGGGCGGATGAGACAAACCATGAATGAATGAGCGGGCGGAACCGCGGAACCAACCATGAATGAGCGGGCGGATGAATGAGCGG GCGGATGAGACAAACCATGAAACCATGAGCGGATGAATGAATGAGCGGGCGGATGAGACAAACCATGAATGAGACAAACCATGAAACCATGAGCGGATGAATGAATGAGCG GGCGGATGAGACAAACCATGAATGAGACAAACCATGAAACCATGAGCGGATGAATGAATGAGCGGGCGGATGAGACAAACCATGAATGAATGAGCGGGCGGAACCGCGGAA CCAACCATGAATGAGCGGGCGGATGAATGAGCGGGCGGATGAATGAGCGGGCGGAACCGCGGAACCAACCATGAATGAGCGGGCGGATGAATGAGCGGGCGGATGAGACAA ACCATGAAACCATGAGCGGATGAATGAATGAGCGGGCGGATGAGACAAACCATGAATGAATGAGCGGGCGGATGAGACAAACCATGAAACCGCGGAACCAACCATGAGACA AACCATGAATGAGACAAACCATGAATGAGACAAACCATGAAACCGCGGAACCAACCAACCGCGGAACCAACCATGAGACAAACCATGAAACCATGAGCGGATGAATGAATG AGCGGGCGGATGAGACAAACCATGA 
Fig. 1 Legend. Context-free L-System [(Axiom: A) A ATGA, C GCGG, T GACA, G AACC] derived $1024 \mathrm{bp}$ sequence from olfactory receptor OR1F1 sequence (ATGAGACAAACCATGA) [http://genome.weizmann.ac.il/cgibin/horde/showGene.pl?key=symbol\&value=oR $1 \mathrm{~F} 1$ ]

Fig. 2

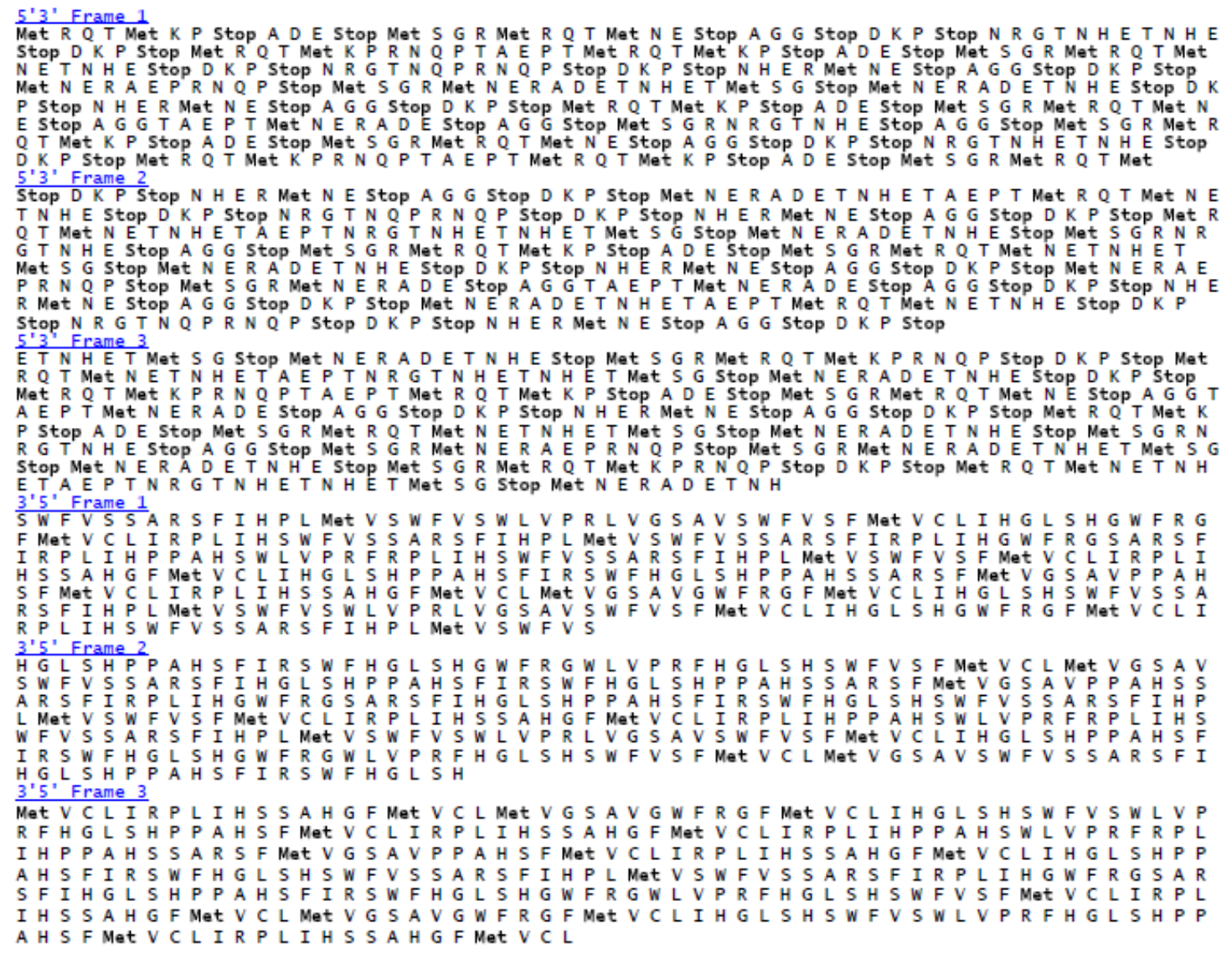

Fig. 2. Legend. Expasy [http://expasy.org/tools/dna.html] generated six reading frames from Fig. 1 show that there are three ORFs in the $3^{\prime} 5^{\prime}$ direction.

As DNA synthesisoccurs in 5' 3' direction therefore we made complementary sequence of Fig. 1 as shown in Fig. 3. We then generated 1024 bp sequence from another L-System [(Axiom: A) A TACT, C CTGT, T TTGG, G TACT] which isthe extreme 5'end of Fig. 3.

TACTCTGTTTGGTACTTTGGTACTCGCCTACTTACTTACTCGCCCGCCTACTCTGTTTGGTACTTACTTACTCGCCCGCCTACTCTGTTTGGTACTTTGGCGCCTTGGTTG GTACTCTGTTTGGTACTTACTCTGTTTGGTACTTACTCTGTTTGGTACTTTGGCGCCTTGGTTGGTTGGCGCCTTGGTTGGTACTCTGTTTGGTACTTTGGTACTCGCCTA CTTACTTACTCGCCCGCCTACTCTGTTTGGTACTTACTCTGTTTGGTACTTACTCTGTTTGGTACTTTGGCGCCTTGGTTGGTTGGCGCCTTGGTTGGTACTCTGTTTGGT ACTTTGGTACTCGCCTACTTACTTACTCGCCCGCCTACTCTGTTTGGTACTTACTTACTCGCCCGCCTTGGCGCCTTGGTTGGTACTTACTCGCCCGCCTACTTACTCGCC CGCCTACTCTGTTTGGTACTTTGGTACTCGCCTACTTACTTACTCGCCCGCCTACTCTGTTTGGTACTTACTCTGTTTGGTACTTTGGTACTCGCCTACTTACTTACTCGC CCGCCTACTCTGTTTGGTACTTACTCTGTTTGGTACTTTGGTACTCGCCTACTTACTTACTCGCCCGCCTACTCTGTTTGGTACTTACTTACTCGCCCGCCTTGGCGCCTT GGTTGGTACTTACTCGCCCGCCTACTTACTCGCCCGCCTACTTACTCGCCCGCCTTGGCGCCTTGGTTGGTACTTACTCGCCCGCCTACTTACTCGCCCGCCTACTCTGTT TGGTACTTTGGTACTCGCCTACTTACTTACTCGCCCGCCTACTCTGTTTGGTACTTACTTACTCGCCCGCCTACTCTGTTTGGTACTTTGGCGCCTTGGTTGGTACTCTGT TTGGTACTTACTCTGTTTGGTACTTACTCTGTTTGGTACTTTGGCGCCTTGGTTGGTTGGCGCCTTGGTTGGTACTCTGTTTGGTACTTTGGTACTCGCCTACTTACTTAC TCGCCCGCCTACTCTGTTTGGTACT 
Fig. 3 Legend. The complementary strand of Fig. 1 in the 5' 3 ' direction.

This sequence when conceptuallytranslated and itgave rise to three oRFs in $5^{\prime} 3^{\prime}$ direction (Fig. 4 ).

Fig. 4

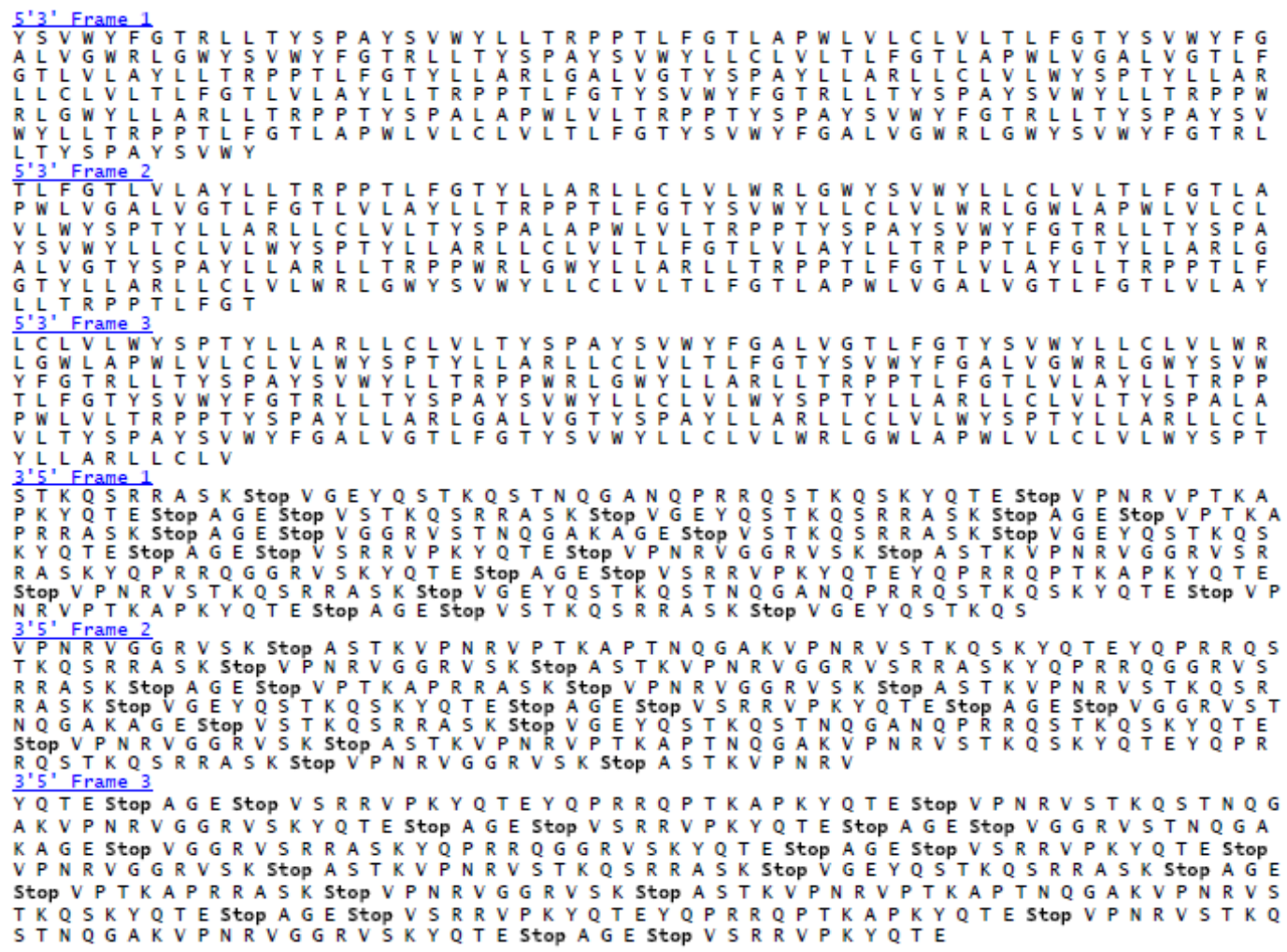

Fig. 4 Legend. Expasy generated sixreading frames from Fig. 3 show that there are three ORFs in the $5^{\prime} 3^{\prime}$ direction.

Fig. 5 


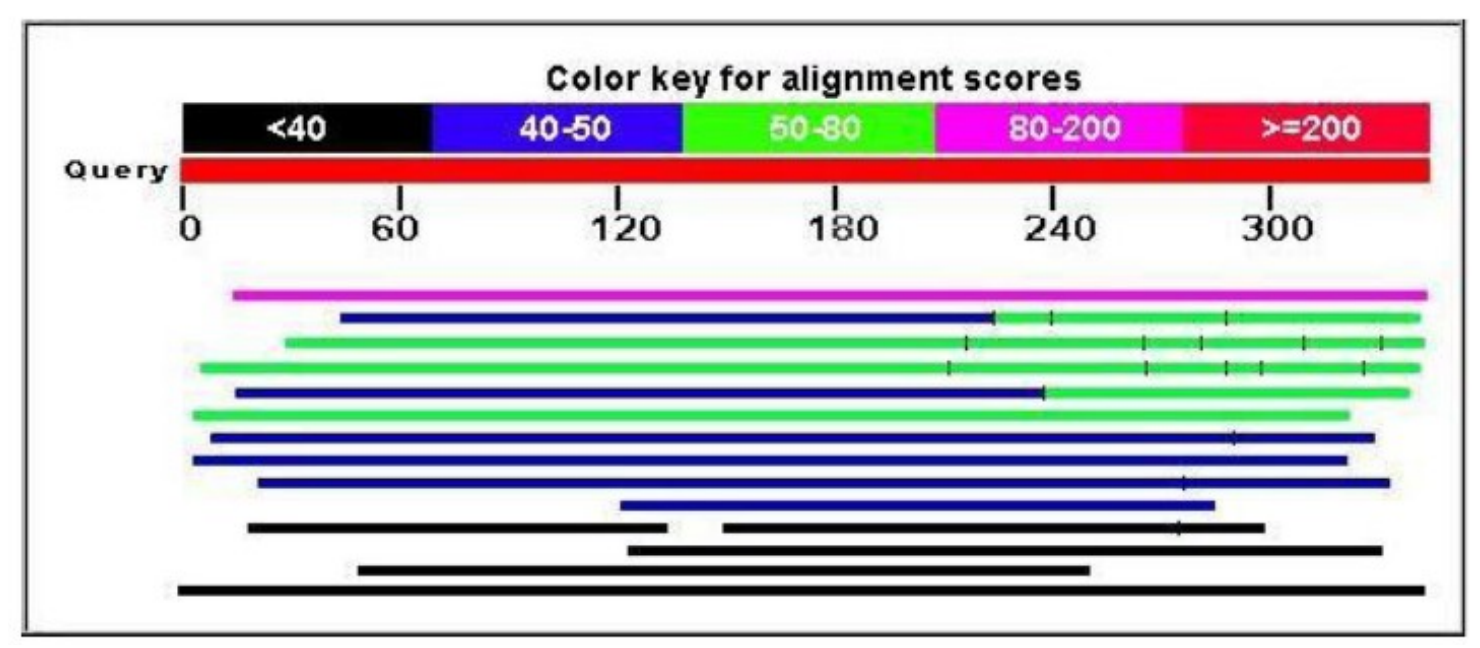

Fig. 5. Legend: FirstORF of Fig. 4 gives $80 \%-200 \%$ homology with a protein domain of nearly 350 amino acids long. This protein has been classified by NCBI as a hypotheticalprotein (1081 aa long) from Gray short-tailed opossum, Monodelphis domestica. Similarlyother two ORFs also exhibited high homology with other organisms (data not shown).

The extreme $5^{\prime}$ end sequence in Fig. 3 was again used as a template (like Fig.1) to generate 1024 bp sequence with following production rule [(Axiom: A) A TTGG, C TTGG, T TACT, G TACT]. On carefulobservation itisclear the production rule of $\mathrm{A}$ and $\mathrm{C}$ are same as well as $\mathrm{T}$ and G. As expected the L-System produced was found to be a highly repetitiveDNA sequence (Fig.6).

Fig. 6

TTGGTTGGTACTTACTTTGGTTGGTACTTACTTTGGTACTCTGTTTGGTTGGTACTCTGTTTGGTTGGTTGGTACTTACTTTGGTTGGTACTTACTTTGGTACTCTGTTTG GTTGGTACTCTGTTTGGTTGGTTGGTACTTACTTTGGTACTCTGTTTGGCTGTTTGGTACTTTGGTTGGTTGGTACTTACTTTGGTTGGTACTTACTTTGGTACTCTGTTI GGCTGTTTGGTACTTTGGTTGGTTGGTACTTACTTTGGTTGGTACTTACTTTGGTTGGTACTTACTTTGGTACTCTGTTTGGTTGGTACTCTGTTTGGTTGGTTGGTACTT ACTTTGGTACTCTGTTTGGCTGTTTGGTACTTTGGTTGGTTGGTACTTACTCTGTTTGGTACTTTGGTTGGTTGGTACTTACTTTGGTACTCTGTTTGGTTGGTTGGTACT TACTTTGGTTGGTACTTACTTTGGTTGGTACTTACTTTGGTACTCTGTTTGGTTGGTACTCTGTTTGGCTGTTTGGTACTTTGGTTGGTTGGTACTTACTTTGGTACTCTG TTTGGTTGGTTGGTACTTACTTTGGTTGGTACTTACTTTGGTTGGTACTTACTTTGGTACTCTGTTTGGTTGGTACTCTGTTTGGTTGGTTGGTACTTACTTTGGTACTCT GTTTGGCTGTTTGGTACTTTGGTTGGTTGGTACTTACTTTGGTTGGTACTTACTTTGGTTGGTACTTACTTTGGTACTCTGTTTGGTTGGTACTCTGTTTGGTTGGTTGGT ACTTACTTTGGTTGGTACTTACTTTGGTACTCTGTTTGGTTGGTACTCTGTTTGGTTGGTTGGTACTTACTTTGGTTGGTACTTACTTTGGTACTCTGTTTGGTTGGTACT CTGTTTGGTTGGTTGGTACTTACTTTGGTACTCTGTTTGGCTGTTTGGTACTTTGGTTGGTTGGTACTTACTTTGGTTGGTACTTACTTTGGTACTCTGTTTGGCTGTTTG GTACTTTGGTTGGTTGGTACTTACT

Fig. 6 Legend. Repetitive DNA sequence generated following [(Axiom: A) A TTGG, C TTGG, T

TACT, G TACT] which showed no significanthomology when searched in NCBI database.

Therefore L-System generation started with human olfactory receptor DNA OR 1 F 1 has reached its limit. We then blastp-ed the hypothetical protein (1081 aa long) from Gray short-tailed opossum, Monodelphis domestica into NCBI database (Fig. 5 with pink color line) and found that ithas a high level of domain homology with Forkhead box protein E4 of Xenopus laevis [Fig.7]. 
Fig. 7

\begin{tabular}{|c|c|c|}
\hline Query & 735 & $\begin{array}{l}\text { PAAVGAVGGGRRRKRPVQRGKP PFSYIALIAMAIAHSAERRLTLGGIYRFITERFAFYRD } \\
\text { P }\end{array}$ \\
\hline Sbjct & 28 & PEEHNQASGGRRRKRPVQRGKP PYSYIAL IAMA IANS PERKLTL GGIYKFIMERFP FYRE \\
\hline Query & 795 & $\begin{array}{l}\text { NPRKWONS IRHNLTLNDCFVKI PRE P GHP GKGNYWALDPAAQDNFD SGSFLRRRKRFKRS } \\
\text { N +KWONSIRHNLTLNDC FVKIPRE PGHPGKGNYT LDPAA+DMFD+GSFLRRRKRFKR+ }\end{array}$ \\
\hline Sbjct & 88 & NSKKTWQNS IRHNLTLND C FVKI PRE P GHP GKGNYTTLD P AAEDMFD NGS FLRRRKR FKRT \\
\hline
\end{tabular}

This domain was re-blastped to NCBI and we found that this domain is highly conserved amongst a number of organisms who are distantlyrelated in evolution [Fig.8].

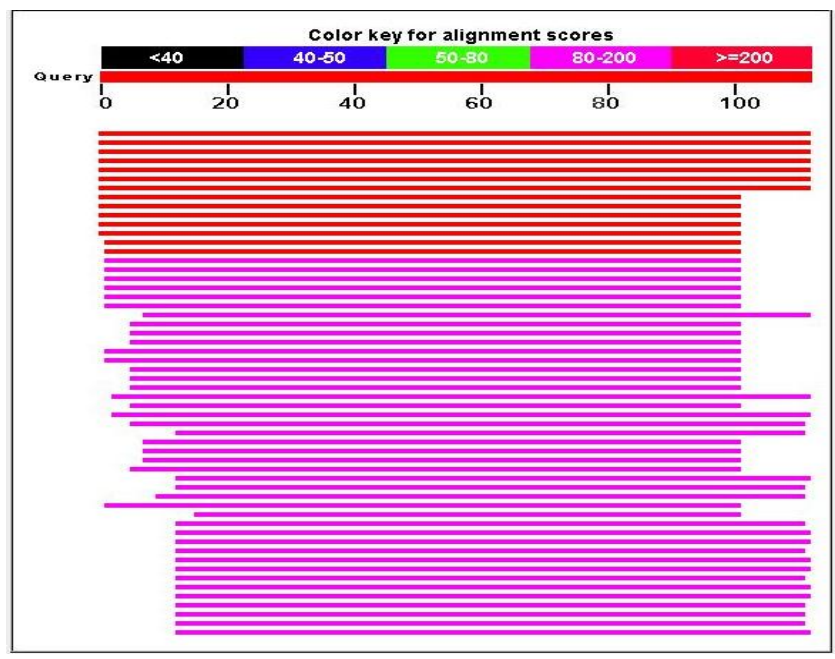

Fig. 8 Legend. Conserved protein domain obtained from Fig. 7 when blastped into NCBI protein database

This result shows that starting with a sixteen base-pair unique stretch of DNA and using LSystem production ruleone can make a conserve protein domain which was hitherto unknown to biologists.We predict that thisishow allconserved proteindomains in livingorganisms have been produced. This mathematical exercise also clearly shows after a conserved protein domain is formed the biological system does not allow any more conserved domain to be formed from the same sequence by producing a repetitivedNA sequence.

Therefore we can conclude that L-System is an important mathematical tool which can be explored to find out the genomic domain shuffling, protein domain formation and repetitive DNA evolution of different organisms. It is tempting to speculate that natural systems might have used this kind of context free L-System derived methodology to generate genomes of different organisms. This method also could be used by syntheticbiologistto find correlation between differentorganismal DNA, proteindomain and finallychange them at will.

ACKNOWLEDGMENTS: This work was supported by the Department of Biotechnology (DBT), New Delhi, grants (BT/PR9050/NNT/28/21/2007 and BT/PR8931/NNT/28/07/2007 to AG) and 
NAIP-ICAR-World Bank grant (Comp-4/C3004/2008-09; Project leader: AG) and ISIplan projects for 2001-2011 to A. G. Authors would like to thank visiting students, Ranita Guha and Shantanav Chakrovorty for theirvaluable inputs in computations.

\section{References}

1. Hassan, S. Sk.,Choudhury, P. P., Pal, A., Brahmachary, R. L. and Goswami, A. (2010) LSystems: A Mathematical Paradigm for Designing Full Length Genes And Genomes. Global Journal of Computer Science and Technology, 10: 119-122, category: I.2.1, J.3, and G.1.0. (Published in June, 2010)

2. Hassan, S. Sk., Choudhury, P. P., Pal, A., Brahmachary, R. L. and Goswami. A. (2010) Designing exons for human olfactory receptor gene subfamilies using a mathematical paradigm. Journal of Biosciences, volume 35, number 3 (to be published as cover page articlein September 2010 issue).

3. Hassan, S. Sk., Choudhury, P. P., Pal, A., Brahmachary, R. L. and Goswami, A. (2010) Combination of L-systems: For Designing Human Olfactory Receptor Pseudo-gene, OR 1 D 3 P. International Journal of Computational Cognition, (Publisher: Yang 's Scientific Research Institute,USA) (Accepted for publication in 2011).

4. Goswami, Arunava, Singh, Rajneesh, Choudhury, Pabitra, and Hassan, Sk. Sarif Hassan. Designing L-Systems formaking three and sixopen reading frames from the leading strand of a single DNA molecule. Available from Nature Precedings <http://hdl.handle.net/10101/npre.2010.4844.1>(2010).

5. Prusinkiewicz, P. and Lindenmayer, A. (1990) in the algorithmic beauty of plants (New York: Springer-Verlag). 